\title{
GRAIN ROTATION AS A MECHANISM OF GRAIN GROWTH IN NANOCRYSTALLINE MATERIALS
}

\author{
Dorel Moldovan ${ }^{1,2}$, Dieter Wolf ${ }^{1}$, Simon R. Phillpot ${ }^{1}$ and Andrew J. Haslam ${ }^{1}$ \\ ${ }^{1}$ Materials Science Division, Argonne National Laboratory, Argonne, Illinois 60439, USA. ${ }^{2}$ Forschungszentrum \\ Karlsruhe, D-76021 Karlsruhe, Germany
}

\begin{abstract}
Grain-boundary (GB) properties in a polycrystalline system are generally anisotropic; in particular, both the GB energy and mobility depend on the GB misorientation. Moreover, in nanocrystalline materials, in which the grain size is less than $100 \mathrm{~nm}$, grain rotations leading to the coalescence of neighboring grains via elimination of the common GB between them may provide a new mechanism for grain growth. Here we investigate the combined effect of curvature-driven GB migration and grain-rotation grain-coalescence on the kinetics, topology and morphology of grain growth. A stochastic velocityMonte-Carlo algorithm based on a variational formulation for the dissipated power is implemented. The presence of both growth mechanisms introduces a physical length scale $\mathrm{R}_{\mathrm{C}}$ into the system, enabling the growth process to be characterized by two regimes. If the average grain size is smaller than $\mathrm{R}_{\mathrm{C}}$, grain growth is dominated by the grain-rotation-coalescence mechanism. By contrast, if the average grain size is greater than $\mathrm{R}_{\mathrm{C}}$, growth is dominated by curvature-driven GB migration. The values of the growth exponents, different for the two growth regimes and different from a system with isotropic GB properties, are rationalized in terms of the detailed growth mechanism and the continuous change of the fraction of low-angle GBs in the system. An extended von Neumann-Mullins relation based on averaged GB properties is proposed and verified.
\end{abstract}

Key words: grain growth, grain rotation, grain coalescence, nanocrystalline materials

\section{INTRODUCTION}

Grain growth is the process by which the average grain size in a polycrystalline material increases in time. The significance of this process comes from the profound influences of the grain size and grain-size distribution on a wide range of properties of polycrystalline materials. Grain size is a key parameter in sintered ceramics, metal and alloy microstructures and usually has to be controlled during thermomechanical processing in order to ensure optimization of mechanical properties. In the classical picture, grain growth takes place via the migration of the grain boundaries (GBs) towards their centers of curvature [1], the driving force being provided by the decrease in energy associated with the decrease in the length of the GBs. This GB-curvature driven grain growth has been the subject of many theoretical [2-6] and simulation studies [7-16]. Most of the simulations assume that all the GBs have the same energy and mobility, i.e., that the energy and mobility are independent of the GB misorientation.

It is well known that real polycrystalline materials exhibit anisotropic GB properties, i.e., both the GB energy and mobility (and, in fact, all other GB properties) are functions of the crystallographic misorientation across the boundary (see, e.g., $[1,17]$ ). The consequences of including the GB anisotropy were first analyzed in two-dimensional (2D) Potts-model simulations [7,8]. GB anisotropy was subsequently also incorporated into front-tracking models of grain growth (see, e.g., [9-16]). In addition to revealing the topological, structural and kinetic consequences of the GB anisotropy, these simulations have recently [16], led to a generalization of the classical Von Neumann-Mullins law for isotropic systems [18,19]. Although this modified law preserves the basic mathematical form of the original equation, its derivation revealed that, 
due to the local nature of the GB anisotropy, the modified law is valid only in a statisticallyaveraged form.

Grain rotations leading to the coalescence of neighboring grains via elimination of the common GB between them may provide another mechanism for grain growth. Even without complete coalescence, the solid rotation of entire grains may lower the total GB energy in the system because the GB energy depends on the misorientation between the grains. Such rotations have been observed in a number of experiments [20-28]. Hermann et al. [20] and Lojkowski et al. [21] used X-ray diffraction to demonstrate that randomly oriented spheres of silver and copper on single-crystal substrates rotate into specific low-energy orientations; direct observation of the rotation of spheres was reported by Mykura [22] using scanning electron microscopy. Ringer et al. [23], observed the rotation of a precipitate particle to a coherent orientation following the passage of a grain boundary. Harris et al. [24] observed grain rotations in free-standing nanocrystalline thin films of gold with columnar microstructure while Nichols et al. [25] observed complete grain coalescence in in-situ transmission-electron-microscopy studies of grain growth. Other recent experiments on micron grain-sized materials reported the presence of grain rotations in connection with texture formation or evolution in polycrystalline nickel $[26,27]$ and aluminum [28].

Our recent molecular-dynamics (MD) simulations of grain growth in a columnar microstructure of fcc palladium with a grain size of $15 \mathrm{~nm}$ demonstrated that, for this rather small grain size, grain-rotation induced grain coalescence provides an effective mechanism for grain growth [29]. The coordinated rotations of neighboring grains observed in these simulations led to grain coalescence by the elimination of the common GB between them. These simulations also demonstrated an intricate coupling of this mechanism with the conventional, GB-curvature driven mechanism.

To date only a few mesoscale simulation studies [30-33] have focused on grain rotation as a mechanism contributing to grain growth. In the study of Nichols et al. [30,31], grain rotation was viewed simply as a means to lower the internal energy in a two-dimensional microstructure of regular hexagons with anisotropic GB energies; no grain coalescence was considered. Saetre et al. [32,33] simulated grain growth by subgrain rotation in one and two-dimensional systems, focusing entirely on the kinetics and topology of grain coarsening due to the grain-rotationcoalescence mechanism alone.

Several theoretical studies have also dealt with the physical mechanism for grain rotation [3438]. All of these assume that the driving force for rotation is the net torque on a grain arising from the misorientation dependence of the energy of the GBs surrounding it, and assume that the accommodation is by either cooperative GB dislocation motion [34-36] or diffusion [34,37,38]. In particular, building on Raj and Ashby's theory of diffusion-accommodated GB sliding at elevated temperatures [39], we have recently developed a theoretical framework describing the rotation of an arbitrarily shaped grain [38] (for details, see Sec. 2).

In the present paper, we extend our previous studies of the role of GB anisotropy on curvature-driven grain growth [16] and investigate the effects of the presence of both GB migration and grain-rotation grain-coalescence mechanisms on the kinetic, topological and morphological aspects of grain growth. The physical basis for our mesoscale simulations is provided by our recently developed theory of diffusion-accommodated grain rotation [38]. A dimensional analysis reveals that the coupling between GB migration and grain rotation during grain growth introduces a physical length scale, $\mathrm{R}_{\mathrm{c}}$, into the system; this absolute length scale is absent if only one of the two mechanisms is activated. The extremes in which one or the other mechanism dominates may therefore be characterized by two regimes: If the average grain size, $\mathrm{R}$, is smaller than $\mathrm{R}_{\mathrm{c}}$, the effects of grain rotations dominate; for $\mathrm{R}>\mathrm{R}_{\mathrm{c}}$, the conventional mechanism of curvature-driven GB migration dominates. Moreover, we demonstrate that in the 
grain-rotation dominated regime an entirely new mechanism of grain growth occurs. This novel grain-growth mechanism exhibits a corresponding scaling behavior with a growth exponent, which can assume a universal value in certain physically reasonable situations. Finally, we demonstrate how the von Neumann-Mullins type relation based on statistically averaged local grain environments, can be extended to include the effects of grain rotations.

The paper is organized as follows. Section 2 contains a brief description of our simulation approach and the underlying theoretical concepts. In Section 3 we study the growth behavior of a hypothetical model system evolving solely under the effects of grain rotations. In addition a simple stochastic theory explaining the growth exponents is proposed. In Section 4 we analyze the coupled effects due to the simultaneous activation of GB migration and grain rotation. Our conclusions are in Sec. 5.

\section{SIMULATION METHOD}

As the formal basis for our mesoscale simulations we adopt the theoretical approach of Needleman and Rice [40] based on a variational principle for dissipative systems. This principle was originally formulated for GB and surface diffusion in the context of void growth [40] and later was adapted for the simulation of curvature-driven grain growth by Cocks and Gill [14,15]. Their modification describes the rate of power dissipation due to the competition between the reduction in the GB energy and the viscous drag during GB migration. Based on the Cocks and Gill formulation of the functional, Cleri [41] developed a stochastic formulation that enables a velocity Monte-Carlo (VMC) simulation approach using the variational functional as transitionrate generating probability. We have used this approach in a study focusing on grain growth in polycrystalline systems with anisotropic GB properties [16]. The simulation method applied here incorporates the effects of grain rotation into the approach implemented by us earlier [16]. Here we briefly highlight the modifications required to incorporate grain rotation to this approach.

Most importantly, the theoretical basis for the present simulations is provided by the theory of diffusion-accommodated grain rotation developed in Ref. [38]. Considering a columnar microstructure of column height unity, the cumulative torque acting on some grain $i$ with respect to its center of mass is given by $[24,38]$

$$
\tau=\sum_{j} L_{j} \frac{d \gamma_{j}}{d \theta_{j}}
$$

where $L_{j}$ denotes the lengths of the individual GBs with energies $\gamma_{j} . \theta_{j}$ is the misorientation angle across the GB between the grain and some neighboring grain $j$. As observed in our MD simulations, analogous to GB migration, grain rotation is a viscous process; i.e., the angular velocity of the grain, $\omega$, with respect to an axis through its center of mass is given by

$$
\omega=M \tau,
$$

where $M$ is the "rotational mobility" of the grain which depends strongly on the average grain size R. Our theoretical treatment [38] yielded a general expression for the grain-size dependence of $\mathrm{M}$, assuming both GB-diffusion and lattice-diffusion accommodation for the rotation. Consistent with this general theoretical framework, here we use the condensed form emphasizing the grainsize dependence for the rotational mobility given by

$$
M(R)=C / R^{p},
$$


where $p=5$ for accommodation by GB diffusion and $p=4$ for accommodation via lattice diffusion through the grain interiors. $C$ is a physical parameter which depends on the material properties, temperature, the grain shape and the accommodation mechanism [38]. In particular, for GB-diffusion accommodated grain rotation and a regular-hexagonal grain shape, $C=95 \Omega D_{\mathrm{GB}} \delta / \mathrm{kT}$, where $\Omega$ is the atomic volume, $\delta$ the diffusion width of the GBs along the periphery of the grain, $D_{\mathrm{GB}}$ the GB self-diffusion coefficient, $k$ Boltzmann's constant and $T$ the absolute temperature.

The initial structure is generated via a 2D Voronoi construction and periodic border conditions are applied to the simulation cell. Analogous to the meshing in finite-element simulations, minimization of the variational functional using the $\mathrm{VMC}$ algorithm requires discretization of the initial microstructure. Two sets of mesh points (or nodes) are introduced, one consisting of the triple points and the other one discretizing the GBs. Each node is linked to neighboring nodes by straight segments; for example, a triple-point node is connected to three neighboring nodes while a GB node is connected to only two other nodes (one or both of which may be associated with triple points). The introduction of this GB mesh allows GB curvature to be modeled in terms of a series of straight segments. Each grain is assigned an initial orientation with respect to a fixed axis in the plane of the simulation cell; therefore each GB is characterized by the misorientation angle between two neighboring grains. Even in a columnar 2D microstructure the full GB texture covers a large parameter space, consisting of both the relative misorientation of two neighboring grains and the inclination of the boundary plane with respect to a reference direction. For simplicity and for comparison with our previous atomistic simulations of grain growth [29] we assume all the GBs in the system to be $<001>$ tilt boundaries. In practice, to account for the $<001>$ texture of the microstructure, the grain orientations are restricted to the $0-90^{\circ}$ interval, and initial grain orientation angles are assigned randomly within this interval (subject to the constraint that no GB misorientation $\theta$ was less than $1^{\circ}$ ). Because the GBs in this system are $<001>$ tilt boundaries in a fourfold symmetric system, only misorientation angles between 0 and $45^{\circ}$ are unique. How this particular textured microstructure affects our results will be discussed at the end of Section 4.3.

As in our earlier study of anisotropic grain growth [16], we assume the following functional forms for the dependence of the GB energy and GB mobility on the GB misorientation. For the GB energy, we use the Read-Shockley formula [42], empirically extended to high angles [43]:

$$
\gamma(\theta)=\gamma_{\max } \sin (2 \theta)[1-r \ln (\sin (2 \theta))],
$$

where $\gamma_{\max }$ is the plateau value and $r$ is a measure of how steeply the GB-energy rises at small angles. The value of the parameter $r$ fitted for $<001>$ tilt GBs in palladium is $r=0.693$ [29]. For the GB mobility we adopt the $\theta$ dependence proposed by Humphreys [44]:

$$
m(\theta)=m_{\max }\left[1-\exp \left(-B\left(\theta / \theta_{0}\right)^{n}\right)\right],
$$

with the parameters $B=5, n=4$ and $\theta_{0}=20^{0}$ [44]. As described in Section 4, the plateau values $\gamma_{\max }$ and $m_{\max }$ do not need to be specified since our simulations will be carried out in reduced units.

To incorporate grain rotations into the model, additional terms are added to the functional; these describe the competition between the reduction in GB energy due to the rotation and the related energy dissipation during the viscous rotation. In addition to the velocity field, $\{\mathbf{v}\}$, of all the GB and triple-point nodes, the dissipated-power functional, $\Pi(\{\mathbf{v}\},\{\omega\},\{\mathbf{r}\},\{\phi\})$, therefore includes also a set of grain-rotation rates, $\{\omega\}$. As described in our previous study [16], $\{\mathbf{r}\}$ 
represents the set of time-dependent coordinates of the GB and triple-point nodes, while $\{\phi\}$ is the set of grain orientations. This extended functional reads:

$$
\begin{gathered}
\Pi(\{\mathbf{v}\},\{\omega\},\{\mathbf{r}\},\{\phi\})=\sum_{i=1}^{N_{s g m}} \Pi_{m i g}^{i}+\sum_{j=1}^{N_{g}} \Pi_{r o t}^{j}= \\
\sum_{i}^{N_{s g m}}\left[\gamma_{i}\left(\mathbf{v}_{i 1} \cdot \mathbf{s}_{i 1}+\mathbf{v}_{i 2} \cdot \mathbf{s}_{i 2}\right)+\frac{l_{i}}{6 m_{i}}\left[\left(\mathrm{v}_{\mathrm{i} 1}^{\mathrm{n}}\right)^{2}+\left(\mathrm{v}_{\mathrm{i} 2}^{n}\right)^{2}+\left(\mathrm{v}_{\mathrm{i} 1}^{n} \mathrm{v}_{\mathrm{i} 2}^{n}\right)\right]+\sum_{j=1}^{N_{g}}\left[\tau_{j} \omega_{j}+\frac{\omega_{j}^{2}}{2 M_{j}}\right]\right.
\end{gathered}
$$

where the first sum is taken over all the GB segments, $N_{s g m}$, of lengths $1_{i}$ used to represent the discretized GB network; the second sum includes all $N_{g}$ grains in the system (see also Eqs. (1)-(3)).

The subscripts 1 and 2 in the first two terms in Eq. (6) (describing the energy balance during curvature-driven GB migration) indicate that the values of the quantities are taken at the two end points of each segment. $\mathbf{s}_{\mathrm{i} 1}$ and $\mathbf{s}_{\mathrm{i} 2}$ are the tangent unit vectors at the ends of segment $i$, considered to point away from the end of the segment. $\mathbf{v}_{\mathrm{i} 1}$ and $\mathbf{v}_{\mathrm{i} 2}$ are the velocities of the nodes associated with segment $i . \mathrm{v}^{\mathrm{n}}{ }_{1}$ and $\mathrm{v}_{\mathrm{i} 2}$ are the normal components of the velocities at the end points of segment $i . \gamma_{i}$ and $m_{i}$, the GB energy and mobility of segment $i$, depend on the misorientation across the segment.

We use Cleri's velocity Monte-Carlo stochastic approach [41] to find the set of GB-node velocities, $\{\mathbf{v}\}$, which minimizes the variational functional at each time step [16]. The grain angular velocities, $\{\omega\}$, are given by Eq. (2). Also, all GBs are discretized so that the Herring relation for the triple-point angles can be satisfied [45]. This results in the GBs being curved, providing the necessary driving force for GB-curvature-driven grain growth. Only in the special case in which grain growth is solely due to the rotation-coalescence mechanism (see Sec. 4), will straight, non-discretized GBs be considered.
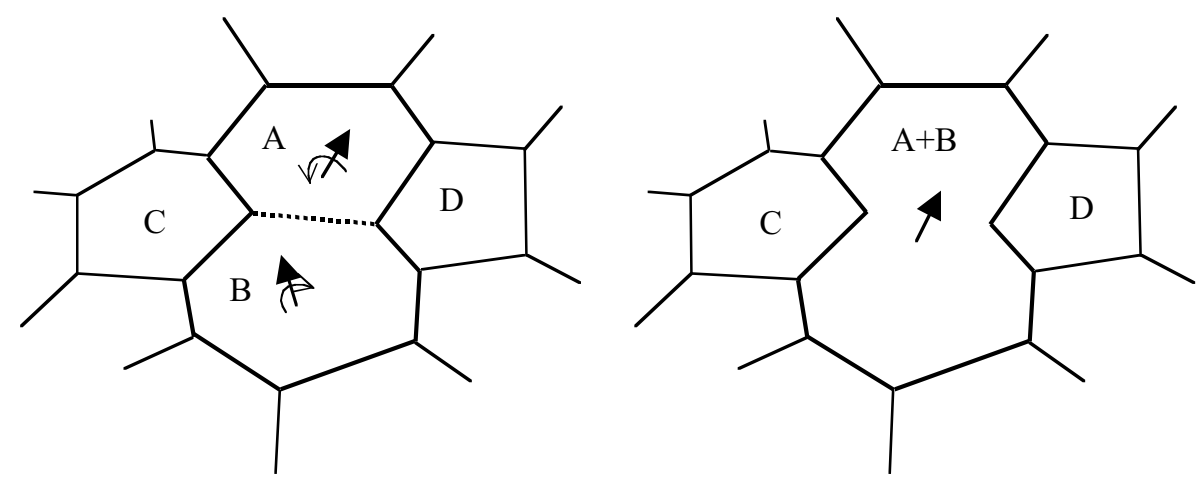

Fig.1. Schematic representation of a grain-coalescence event. In (a), grains A and B sharing a common boundary (dotted line) rotate towards one-another until the GB misorientation vanishes, i.e., the GB disappears, leading to the coalescence of the two grains sketched in (b). As seen in (b), the coalescence gives rise to a topological discontinuity associated with the elimination of two triple junctions. The nature of this discontinuity, and the subsequent relaxation of the microstructure, were observed in our earlier MD simulations of grain growth [29].

In addition to the well-known $\mathrm{T} 1$ and $\mathrm{T} 2$ topological discontinuities associated with neighbor switching and the disappearance of three-sided grains [46], grain coalescence gives rise to the topological discontinuities shown schematically in Fig. 1. In particular, the disappearance of an entire GB also eliminates the two triple junctions at the GB endpoints. In practice, this topological discontinuity is incorporated in our simulations by considering two neighboring 
grains as having coalesced when the misorientation of their common GB is less than $0.5^{\circ}$; at that point, the appropriate topological rearrangements are carried out.

\section{GRAIN GROWTH BY ROTATION-COALESCENCE ALONE}

In a two-dimensional system, grains can rotate about the texture axis in the clockwise or counterclockwise direction. The change of orientation of a grain during rotation will lead to a change in the misorientations and energies of all the GBs surrounding the grain, such that the total energy of all the GBs delimiting the grain will decrease. According to Eq. (1) the GBs with the largest contribution to the cumulative torque are those with the smallest misorientation angle, as they have the largest value of the gradient $\mathrm{d} \gamma(\theta) / \mathrm{d} \theta$. The rate of grain rotation is given by Eq. (2), in which the rotational mobility, $M$, depends strongly on the average grain size, $R$, as illustrated in Eq. (3).

One of the main aims of the current simulations is to examine the effects of grain rotation on the kinetics and topological aspects of grain growth. However, here we focus on the growth in the presence of grain rotation only, while the combined effects of both mechanisms will be investigated in Sec. 4. To explore the effect of the particular functional form of the misorientation dependence of the GB energy, $\gamma(\theta)$ two different functional forms are considered: i) A realistic functional form for the $<001>$ tilt GBs expressed as the extended Read-Shockley formula in eqn. (4). ii) A simplified triangular shape $\gamma(\theta) / \gamma_{\max }=4 \theta / \pi$ for $0<\theta<\pi / 4$ and $\gamma(\theta) / \gamma_{\max }=2-4 \theta / \pi$ for $\pi / 4<\theta<\pi / 2$, which depends on just one parameter, $\gamma_{\max }$, which we set equal to one. Moreover, to better understand the effect of the grain rotational-mobility parameter $\mathrm{p}$ in Eq. (3) on the growth process, we have performed simulations for $\mathrm{p}=3,4$ and 5 employing both of the above mentioned functional forms for $\gamma(\theta)$. While the cases for $p=5$ and $p=4$ have direct physical interpretation in terms of a diffusion-accommodation mechanism [16], the value $p=3$ is primarily of mathematcal interest.

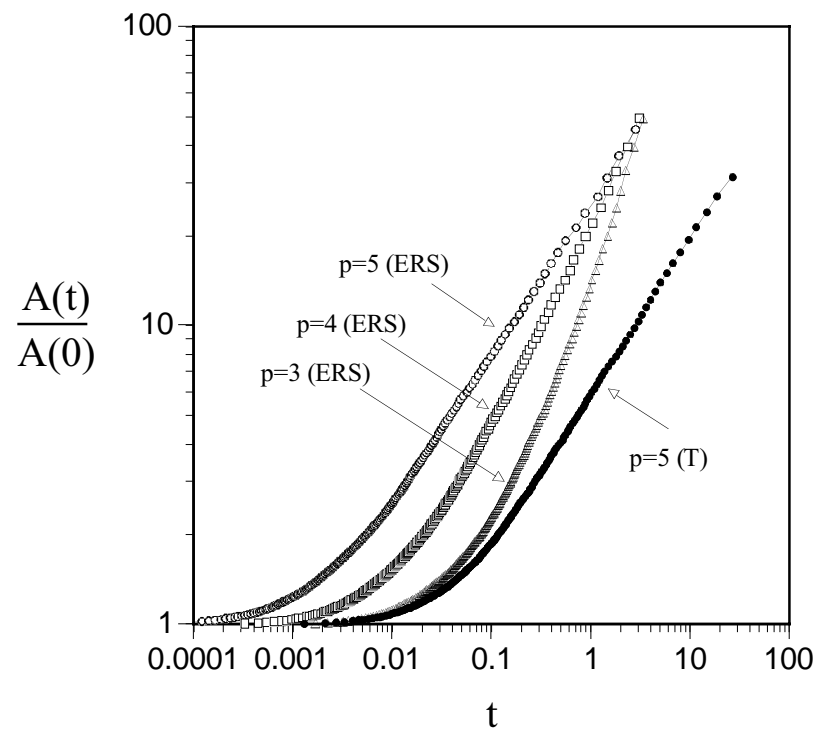

Fig. 2 Time variation of the average grain area (normalized using the initial value) for various values of the parameter p. In these simulations both the extended Read-Shockley (ERS) and triangular (T) formulas are used for the GB energy dependence on the misorientation. 
We first focus onthe growth kinetics. Figure 2 shows the time variation of the average grain area for the systems with various values of the parameter $p$ and for $\gamma(\theta)$ given by both the extended Read-Shockley (Eq. (4)) and triangular formula. We follow the time evolution of the total number of grains from initially 10,000 until only about 300 grains remain. After some transition period, which is different for each value of $p$, the systems reach scaling regimes in which the growth is characterized by power laws (i.e. $\left.A(t) \sim t^{\mathrm{n}}\right)$ with different growth exponents $\mathrm{n}$. Interestingly, the growth kinetics is very similar for the two quite different functional forms of $\gamma(\theta)$. This suggests that the growth exponent assumes a universal value in a similar way to the growth exponent in the systems evolving by curvature-driven GB migration growth in isotropic systems.

A theoretical model predicting the values of the exponent $\mathrm{n}$ for the grain growth by grainrotation induced grain coalescence and based on the mean-field approach, similar to that applied to curvature-driven grain growth by Burke and Turnbull [2], is presented in the Appendix. This predicts that the exponent, $\mathrm{n}$, which under certain conditions is universal depends only on the parameter $\mathrm{p}$ in Eq. (3), i.e., on the specific accommodation mechanism. Table 1 summarizes the simulation findings together with the theoretically predicted values of the growth exponent. One can see that the growth exponents $n=2 /(p-1)$ for both functional forms of $\gamma(\theta)$ are almost the same and in very good agreement with the theoretically predicted exponents given by Eq. (A.8). We should mention here that the value of the parameter q entering Eq. (A.7) and determined from the simulations is indeed very small $(\mathrm{q} \sim 0.02-0.05)$ for both functional forms. Our simulation results, corroborated by our analytical derivation, thus suggest the existence of a new universal coarsening exponent, $\mathrm{n}$, characterizing the growth in $2 \mathrm{D}$ domain structures by the mechanism of grain-rotation induced grain coalescence. This coarsening exponent only depends on the parameter $\mathrm{p}$ characterizing the size dependence of the rotational mobility of the grains.

Table 1. Analytical and simulated values for the coarsening exponents $n$ characterizing, the time dependence of the average grain area, $A(t) \sim t^{n}$, for the system evolving in the presence of grain rotation only. Simulation results are reported for two functional types of the misorientation dependence of the grain boundary energy $\gamma(\theta)$.

\begin{tabular}{cccc}
\hline $\mathrm{p}$ & $\mathrm{n}$ & \multicolumn{2}{c}{$\mathrm{n}$ (Simulation) } \\
\cline { 3 - 4 } & (Eq. (A.8)) & $\begin{array}{c}\gamma(\theta) \text {-extended } \\
\text { Read-Shockley }\end{array}$ & $\begin{array}{c}\gamma(\theta) \text {-triangular } \\
\text { shape }\end{array}$ \\
\hline 5 & 0.50 & 0.50 & 0.51 \\
4 & 0.66 & 0.66 & 0.67 \\
3 & 1.0 & 0.99 & 0.95 \\
\hline
\end{tabular}

Apart from the increase of the average grain size, other important microstructural features, such as the balance between low-angle and high-angle GBs, are modified during growth. This is best characterized by the misorientation-angle distribution function, which gives the fraction of the total number of GBs with a given misorientation. For reference and comparison, in addition to the distributions for the system evolving by rotation coalescence, Fig. 3 shows the distributions for the same system evolving by curvature driven GB migration only. Since the driving force for grain rotation arises mainly from the low-angle GBs, and since each grain-coalescence event eliminates a low-angle GB, for these systems grain rotation significantly changes the content of low- and high-angle GBs during growth: compared with the initial distribution at $t=0$ $(\mathrm{N}=10,000)$, this fraction decreases significantly during growth. This is opposite to what happens in a system evolving by GB migration only for which the fraction of low-angle GBs increases in 
time (see ref. [16]). As also shown in our previous study [16], in a microstructure generated by assigning random uniformly distributed grain orientations the misorientation-angle distribution is independent of misorientation (see the crosses in Fig. 3).

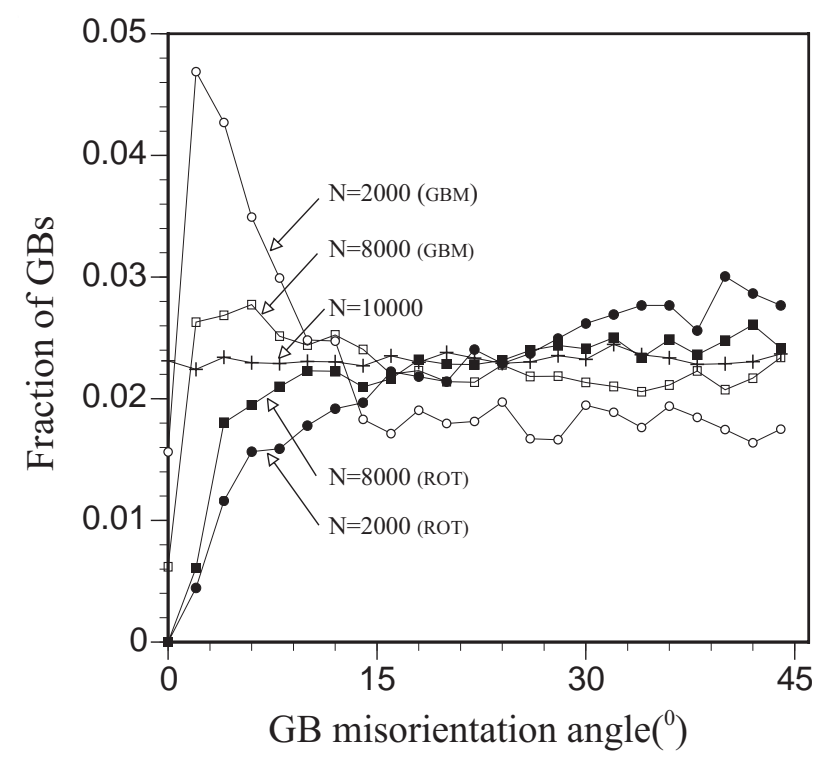

Fig. 3 Comparison of the GB misorientation distribution functions vs. GB misorientation angle for systems evolving in the presence of grain rotation only (ROT) and GB migration (GBM) only at various times; there were initially 10,000 grains.

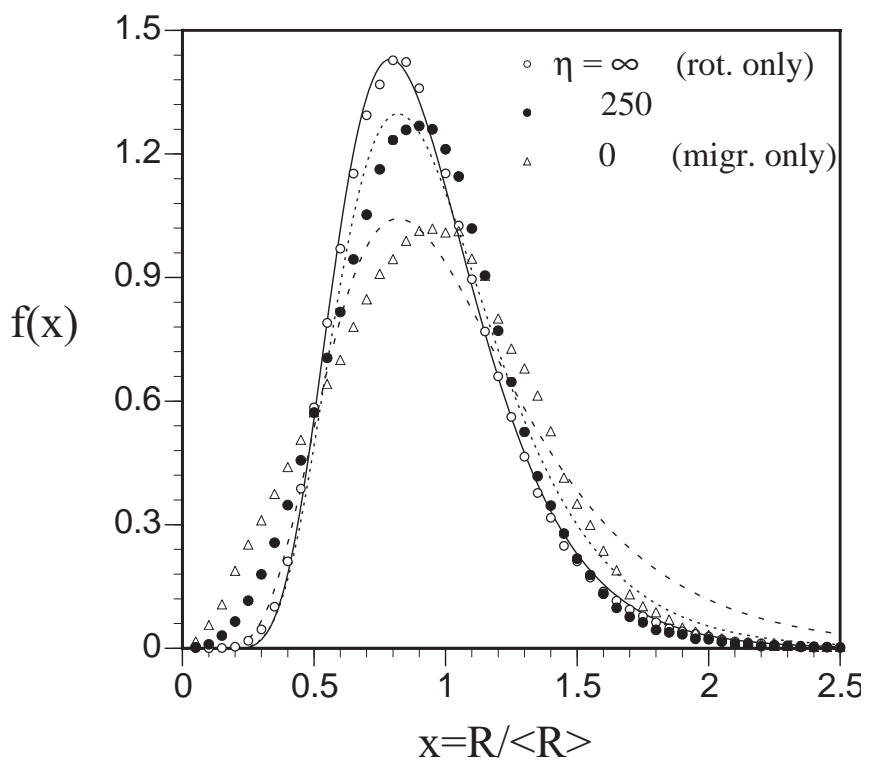

Fig. 4 Grain-size distribution functions vs. reduced grain size, $\mathrm{x}=\mathrm{R} /<\mathrm{R}>$, for three systems evolving in the presence of grain rotation only (o), grain rotation plus GB migration $(\bullet)$ and in the presence of only GB migration ( $\square$ ). For comparison, the log-normal distributions fitted for rotation-coalescence $(-)$, rotation-coalescence plus migration (......) and for GB migration (-----) mechanisms are shown.

The simulations also reveal additional information regarding the grain morphology and the grain-size distribution of the coarsening microstructure. Figure 4 shows the grain-size 
distributions evaluated in the scaling regimes for three systems evolving in the presence grain rotation only, GB migration only and in the presence of both grain rotation and GB migration (the case when both grain rotation and GB migration are present, will be discussed in Sec. 4.3). For comparison, the best-fit log-normal distribution functions for all three distributions are also given. Interestingly, the grain-size distribution function for the system evolving by rotation-coalescence mechanism follows almost perfectly a log-normal distribution; by contrast, in the system evolving by GB migration there is a considerable deviation from the log-normal distribution. The grainrotation coalescence distribution is narrower and has a higher peak value than that for the system evolving by GB migration; it also drops more quickly to zero at small grain sizes and even shows an apparent cut-off at about 0.2 in the reduced grain-size. One can rationalize the differences between the two distribution functions by recalling that under GB migration small grains shrink continuously until they disappear. However, when only grain rotation takes place, there is no mechanism by which a grain can decrease its size: indeed, a grain may only increase its size by discrete coalescence events with its neighboring grains.

\section{GRAIN GROWTH BY SIMULTANEOUS GB MIGRATION AND GRAIN ROTATION}

We now consider growth under the combined effects of both grain rotation and GB migration. As we show the simultaneous presence of both GB migration and grain rotation introduces a physical length scale into the system, in the form of a physical grain size, $R_{\mathrm{c}}$; this absolute length scale is absent if only one of the two mechanisms is present. We estimate the value of this physical length scale by using a dimensional analysis.

\subsection{Dimensional analysis of characteristic length and time scale}

For the purpose of this dimensional analysis, we consider a single representative term in Eq. (6). In the presence of both GB migration and grain rotation, this term has the form, $\Pi=\Pi_{\text {mig }}+$ $\Pi_{\text {rot }}=\left[\gamma_{\mathrm{v}}+\left(\mathrm{v}^{2} / \mathrm{m}\right) 1\right]+\left[\tau \omega+\left(\omega^{2} / \mathrm{M}\right)\right]$. To put $\Pi$ into dimensionless form we choose to express the GB energy, $\gamma$, in units of $\gamma_{\max }$, the GB mobility, $m$ in units of $m_{\max }$, the length, $l$, of a GB segment in units of the initial average grain radius, $\mathrm{R}_{0}$ and the time, $t$, in units of $\mathrm{t}_{0}=\mathrm{R}_{0}{ }^{2} / \gamma_{\max } \mathrm{m}_{\max }$. Having chosen these, the representation in reduced units for the other physical quantities of interest are: $\mathrm{v}_{0}=\gamma_{\max } \mathrm{m}_{\max } / \mathrm{R}_{0}$ for the node velocity $\mathrm{v}, \omega_{0}=\gamma_{\max } \mathrm{m}_{\max } / \mathrm{R}_{0}^{2}$ for the angular velocity $\omega$, and $\tau_{0}=\mathrm{R}_{0} \gamma_{\max }$ for the torque $\tau$. This choice allows us to define the dimensionless variables: $\gamma^{*}=\gamma / \gamma_{\max }$, $\mathrm{m}^{*}=\mathrm{m} / \mathrm{m}_{\max }, \mathrm{R}^{*}=\mathrm{R} / \mathrm{R}_{0}, \mathrm{v}^{*}=\mathrm{v} / \mathrm{v}_{0}, \tau^{*}=\tau / \tau_{0}$ and $\omega^{*}=\omega / \omega_{0}$. Using these, the above sum of the two representative variational-functional terms can be written in dimensionless form as follows:

$$
\Pi^{*}=\frac{\Pi}{\Pi_{0}}=\left[\gamma^{*} v^{*}+\frac{v^{* 2}}{m^{*}} l *\right]+\left[\tau * \omega^{*}+\frac{1}{\eta} \frac{\omega^{* 2}}{M^{*}}\right],
$$

where $\Pi_{0}=\left(\gamma_{\max }{ }^{2} m_{\max }\right) / \mathrm{R}_{0}$ and $\mathrm{M}^{*}=\left(\mathrm{R}_{0} / \mathrm{R}\right)^{\mathrm{p}}$. It is important to notice that we have introduced the dimensionless parameter $\eta=\left(C / m_{\max } R_{0}^{p-3}\right)$; i.e., the time evolution of the microstructure described by this functional depends on the actual value of the parameter $\eta$. The parameter $\eta$ can be written in the equivalent forms:

$$
\eta=\frac{C}{m_{\max } R_{0}^{p-3}}=\left(\frac{R_{c}}{R_{0}}\right)^{p-3}
$$


where

$$
R_{c}=\left(\frac{C}{m_{\max }}\right)^{\frac{1}{p-3}}
$$

is a characteristic physical length scale. Note that for $\mathrm{p}=5, \eta \sim \mathrm{R}_{\mathrm{c}}{ }^{2}$, whereas for $\mathrm{p}=4, \eta \sim \mathrm{R}_{\mathrm{c}}$.

Equation (8) illustrates that $\eta$ may be viewed as the ratio of two lengths: the average initial grain size, $\mathrm{R}_{0}$, which depends on the microstructure, and the physical length scale $\mathrm{R}_{\mathrm{c}}$ determined solely by the material properties and temperature, i.e., the GB mobility, $m_{\max }$, and the materials constant $\mathrm{C}$. Thus, when $\mathrm{R}_{0}>>\mathrm{R}_{\mathrm{c}}, \eta$ is small and the growth is GB-migration dominated, whereas when $\mathrm{R}_{0},<<\mathrm{R}_{\mathrm{c}}, \eta$ is large and the growth is grain-rotation dominated. For a hexagonal grain shape and assuming GB-diffusion as the accommodation mechanism for grain rotation, i.e., $\mathrm{p}=5$ in Eq. (3), $R_{c}=\left(C / m_{\max }\right)^{1 / 2}$.

Up to a numerical factor of the order unity $\mathrm{R}_{\mathrm{c}}$ is the same as $d_{\mathrm{c}}$, the critical grain size, where the crossover from rotation-coalescence to GB-migration dominated grain growth occurs [29]. Using a different line of reasoning, we have estimated $d_{\mathrm{c}}$ in a previous study [29]. In particular, using data for $\mathrm{Pd}$ at $1400 \mathrm{~K}$, i.e. just below the melting point, we estimated that $\mathrm{d}_{\mathrm{c}}$ is $\sim 3.2 \mathrm{~nm}$. We interpreted this result as an indication that, in a pure material, grain rotations only contribute to grain growth if the grain size is of the nanometer dimensions [29].

The parameter $\eta$ is analogous to the Reynolds number in the analysis of the Navier-Stokes equation describing the incompressible flow of viscous fluids in hydrodynamics. The Reynolds number characterizes the relative importance of inertial and viscous forces; at a certain value of the Reynolds number the flow regime changes from laminar to turbulent flow. It is also important to recall that the Reynolds number is used to characterize the flow similarity; for example, the flows around two geometrically similar bodies will be identical (in dimensionless variables) if the Reynolds numbers for the two bodies are the same. Introduction of the parameter $\eta$ has similar implications. In particular, a certain value of $\eta$ marks the transition from GB-migrationdominated to rotation-dominated growth and can be used to study similarity of the growth.

In the context of the physical interpretation of the parameter $\eta$, there are two limiting cases: $\eta$ $=\infty$ characterizes the system in the presence of grain rotation only, whereas $\eta=0$ characterizes the system in the presence of GB migration alone.

\subsection{Growth law and the crossover regime}

We first analyze the growth law to determine the range of $\eta$ values for which grain rotation and GB migration compete on an equal basis. We then perform a detailed analysis for one particular value of $\eta(\eta=250)$ in this crossover regime.

As shown in Figs. 5(a), and 5(b) the value of the growth exponent, $n$, depends on the parameter $\eta$. Specifically, we find that for $\eta<10$, the growth exponent is $n \approx 0.71$, which is the same as the value for the system in the presence of GB-migration only (see ref. [16]). Similarly, for $\eta>3000$ we find $n \approx 0.50$, i.e., the same as the value obtained in the presence of growth by grain rotation-coalescence only (see Fig. 2). In the range $10<\eta<3,000, \mathrm{n}$ decreases smoothly (and approximately exponentially with $\eta$ ) from $n=0.71$ to $n=0.5$. Figure 5(b) reveals that a value of $\eta \approx 250$ sets the system in the middle of the crossover interval.

We can rewrite Eq. (8) as $R_{0}=R_{\mathrm{C}} / \eta^{1 / 2}$; i.e., one can have the alternate view of the crossover regime in terms of the initial average grain size $R_{0}$. We find that the GB-migration dominated regime is obtained for grain sizes larger than $R_{0}>0.33 R_{\mathrm{c}}(\eta<10)$, while for $R_{0}<0.018 R_{\mathrm{c}}(\eta>$ $3,000)$ the system is in the grain-rotation dominated regime. When considering these values one 
should bear in mind that, provided the growth process is followed over a long time such that significant changes of the average grain size occur, a continuous change from rotation-dominated to migration-dominated growth is obtained. In our simulations for growth in the scaling regime (evolution from 5000 to 500 grains) the average grain size increases by roughly a factor of three.

(a)

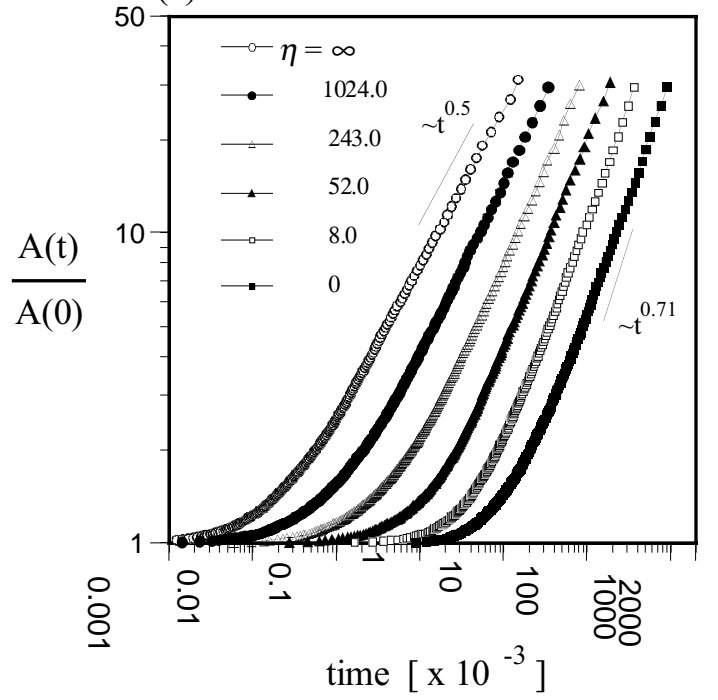

(b)

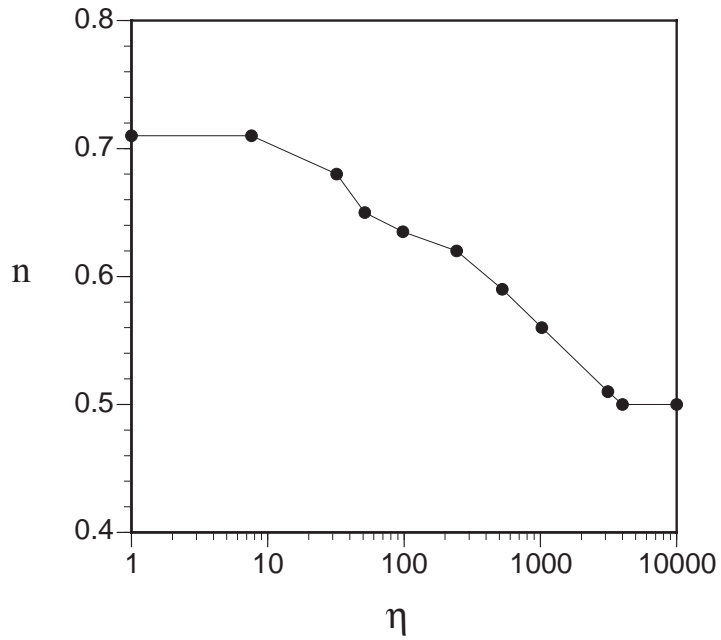

Fig. 5 (a) Time variation of the average grain area (normalized using the initial value) for systems evolving in the presence of both grain rotation and GB migration (i.e. various values of the parameter $\eta$ ). (b) $\eta$ dependence of the grain-growth exponent $n$.

Figure 6 shows four snapshots of evolving microstructures with different values of $\eta$ ranging between 0 and $\infty$; all snapshots contain about 700 grains evolved from initially 10,000 grains. It is interesting to see that in the presence of grain rotation only $(\eta=\infty$, see Fig. 6(a)), most of the grains are relatively elongated. In addition, close inspection of this microstructure indicates the presence of a relatively small number of small grains. One can therefore anticipate a narrower grain-size distribution compared with the cases when GB migration is present. By contrast, for $\eta$ $=0$ (see Fig. 6(b)) there are no significantly elongated grains in the microstructure. In this case the microstructure has an overall resemblance to that of isotropic grain growth (i.e., soap froth, see Fig. 6(d)). However, there are some differences due mainly to the triple-point angles deviating from $120^{\circ}$, which is a consequence of the presence of both low-angle and high-angle GBs in the system. The morphology of the microstructure is quite different when both GB migration and grain rotation are present (see the system for $\eta=250$ in Fig. 6(c)). This microstructure exhibits characteristics of both microstructures in Figs. 6(a) and 6(b). For example, one can see the presence of some elongated grains, the signature of grain rotation; also, the presence of some relatively small grains compared with the average, reflecting a broader distribution of the grain sizes, is a characteristic of GB migration only (see Fig. 6(b)). We find that in the presence of GB migration (both with and without grain rotation), the dihedral angles between boundaries at triple junctions quickly assume their equilibrium values and the Herring relation [45] is satisfied. 

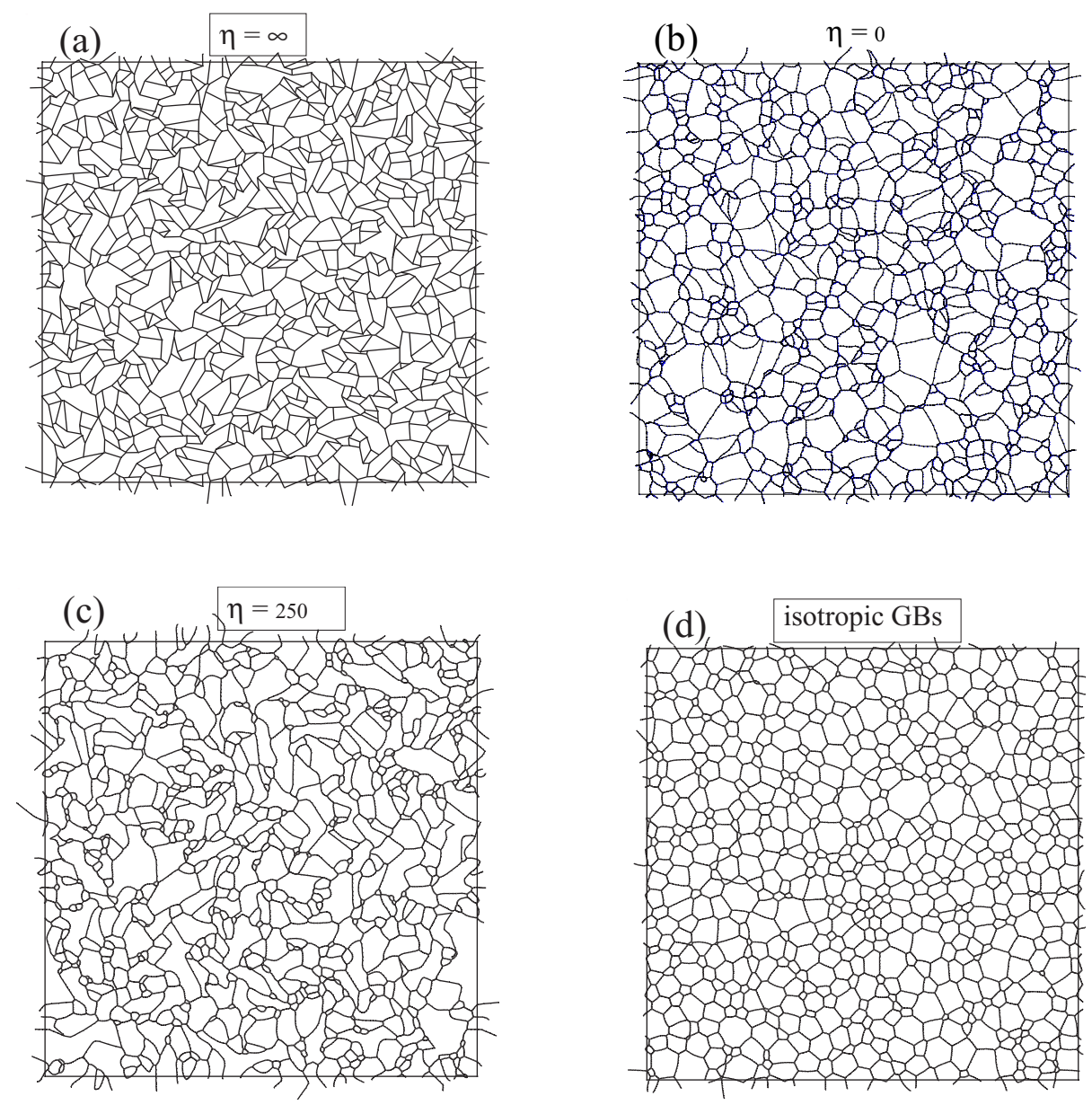

Fig. 6 Four typical snapshots of the microstructure when only about 700 (from initially 10,000) grains are left in a system evolving under various growth conditions. (a) grain rotation only $(\eta=\infty)$. (For this system the straight GB model was used in the simulations.); (b) Curvature- driven GB migration only ( $\eta=0$ ); (c) Simultaneous grain rotation and GB migration $(\eta=250)$ and (d) GB migration only in a system with isotropic GB properties.

\subsection{Distribution functions}

Figure 4 shows that the presence of both grain rotation and GB migration $(\eta=250)$ leads to a widening of the grain-size distribution compared with the case when only rotation is present and a shift of the peak positions towards larger reduced grain sizes. Moreover, GB migration tends to eliminate the cut-off threshold $x_{c}$ at small grain sizes, i.e., grains which are much smaller than the average start to be present more frequently in these systems. One can understand this by recalling that under GB migration small grains will shrink continuously until they disappear (by a T2 event or by a combination of $\mathrm{T} 1$ and $\mathrm{T} 2$ events). However, when only grain rotation takes place, there is no mechanism by which a grain can decrease its size. Rather, a grain may only increase its size by discrete coalescence events with its neighboring grains. Moreover, since the rotational mobility depends so strongly on the average grain size (see Eq. (3)), small grains will be more likely to undergo coalescence events, and therefore grow faster, than larger ones. 


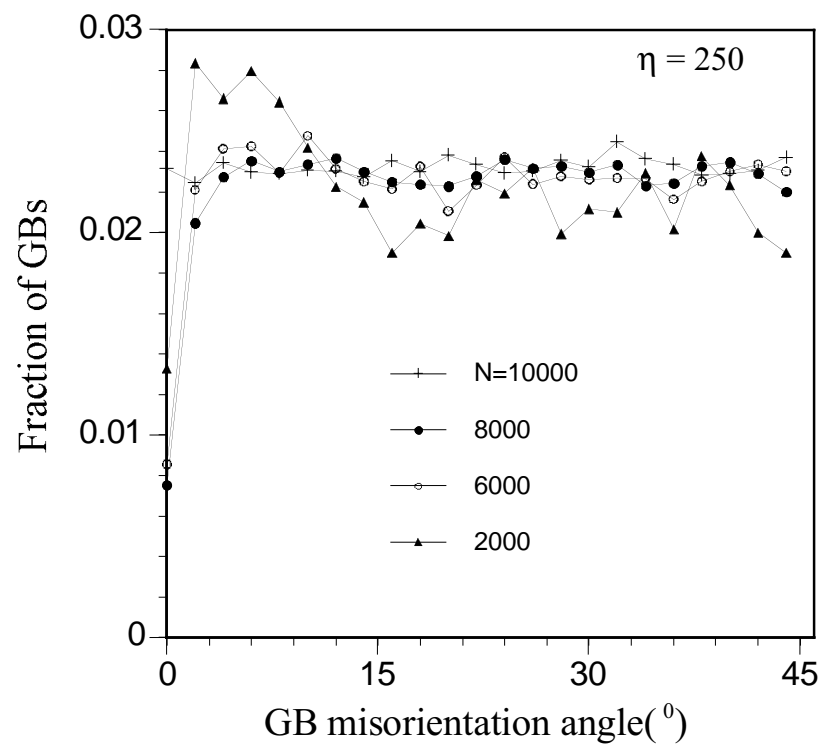

Fig. 7 GB misorientation distribution functions vs. GB misorientation angle for $\eta=250$. The distribution functions are plotted at various times; initially the system contained 10,000 grains.

Finally, we mention that there is no consensus on the precise form of the grain-size distribution of a microstructure evolving under curvature-driven grain growth. While experimental data suggest that a log-normal distribution function is appropriate, neither theoretical models nor simulation studies (see, e.g., Fig. 4) have reproduced such a distribution. Therefore, the deviations from the log-normal distribution are not surprising.

As shown in Fig. 3, during the growth by grain rotation-coalescence alone, the fraction of low-angle GBs decreases significantly, while for curvature-driven GB migration alone the opposite trend is observed. Thus, it is interesting to see the evolution when both are present. For $\eta$ $=250$, Fig. 7 shows that initially the fraction of low-angle GBs decreases slowly until about 8,000 grains are left in the system. As the number of grains continues to decrease, the fraction of lowangle GBs starts to increase slowly. By the time there are 6,000 grains left in the system, the misorientation distribution function is almost the same as the one at the start when 10,000 were present. Further grain growth leads to a small increase of the fraction of low-angle GBs. This time dependence illustrates the delicate balance between the mechanisms of GB migration and grain rotation.

In summary, in the grain-growth regime dominated by GB-migration the main driving force is given by the high-angle GBs with their higher energies and mobilities (see [16]); by contrast for growth in the grain-rotation dominated regime the low-angle GBs, with their higher $(\mathrm{d} \gamma / \mathrm{d} \theta)$, are more important. One should also notice that in each regime the fraction of the GBs that contributes most to the growth diminishes (see Figs. 3 and 7) as the grain growth progresses. As mentioned in Section 2 in the columnar 2D $<001>$ textured microstructure considered in these simulations, the GB misorientations are restricted to $0-45^{\circ}$ and the probability of any particular GB misorientation in the $0-45^{\circ}$ is constant. Consistent with this, $45 \%$ of all the GBs in the system are misoriented by $20^{\circ}$ or less and all of these will experience energy gradients causing rotation. A quite different misorientation distribution function characterizes a fully $3 \mathrm{D}$ microstructure with random orientations of the grains. This was calculated first by Mackenzie and Thomson [47] and later demonstrated experimentally by Samajadar and Doherty [48]. In the 3D cubic crystal structure, relevant to $2 \mathrm{D}$ growth in thin film columnar structures as well as in fully $3 \mathrm{D}$ structures, 
only about $4 \%$ of the GBs are misoriented by less than $20^{\circ}$. Hence far fewer GBs are close to a zero angle misorientation. Consequently, one expects that rotation effects will be less important in a fully $3 \mathrm{D}$ cubic crystal structure. Moreover, the crossover grain size $\mathrm{R}_{\mathrm{c}}$ to the evolution of the microstructure under partial or full rotation control will be shifted to even smaller grain sizes.

\subsection{Extended von Neumann-Mullins relation}

For a system with isotropic GB properties evolving by curvature-driven GB migration, von Neumann [18] derived the key equation,

$$
\frac{d A_{n}}{d t}=\frac{\pi \gamma m}{3}(n-6)
$$

relating the rate of area change of a grain to its number of sides, $n$, and to the product of the GB energy $\gamma$ and mobility $m$. Eq. (10), known as the von Neumann-Mullins (VNM) relation, allows a simplified description of a coarsening network in an isotropic system in terms of only two variables: the area, $A_{\mathrm{n}}$ and the topological class, $n$, i.e., the number of sides of each grain. According to Eq. (10), the rate of area change for each grain is independent of the boundary shapes and of the rates of growth of the neighboring grains.

Focusing on the effect of GB anisotropy on grain-growth in a previous study [16], we investigated the adherence of curvature-driven grain growth to an extended, averaged VNM relation:

$$
\frac{d<A_{n}(t)>}{d t}=<\gamma_{n} m_{n}>\left(\pi-<\theta_{n}>\right)\left[n-\frac{2 \pi}{\pi-<\theta>}\right],
$$

where $<\gamma_{n} m_{n}>$ is the average value of $\gamma m$ for the GBs surrounding the grains of topological class $n$, and $\left\langle\theta_{n}\right\rangle$ is the average value for the triple-point angles of the $n$-sided grains; $<\ldots>$ indicates an average over all grains with $n$ sides. One can see that Eq. (11) reduces to the VNM relation for isotropic systems by simply substituting $\left\langle\theta_{n}>=2 \pi / 3\right.$ and $<\gamma_{n} m_{n}>=\gamma m$.

We point out that Eq. (11) requires input from simulation, specifically the averaged quantities $\left\langle\gamma_{n} m_{n}\right\rangle$ and $\left\langle\theta_{n}\right\rangle$, whereas the VNM relation requires no simulation input. Our simulations revealed that, although the growth is considerably more complex in a system with anisotropic GB properties, the growth process can still be rationalized in terms of groups of grains belonging to the same topological class [16].

In the presence of both GB migration and grain rotation, two processes contribute to the change of the grain area. While GB migration leads to a continuous and smooth change of the area, change due to the grain-rotation-coalescence mechanism is sudden and discrete. This discrete change is described in the Appendix.

Figure 8 shows the time evolution, over a relatively short period of time, of the average area, $<A_{n}(t)>-<A_{n}(0)>$, of $n$-sided grains in the scaling regime for the system with $\eta=250$. One should notice that only grains that do not change their number of sides are considered when calculating $\left\langle A_{n}(t)\right\rangle$. Although the rate of area change of individual grains in a topological class varies, the rate of change of the average area of the grains in each topological class is approximately constant. Figure 8 reveals that the average area of a six-sided grain does not change; by contrast, the average areas of grains with $n<6$ decrease and those with $n>6$ increase. Moreover, the rates of average area change do not increase linearly with $n$, by contrast with the prediction from the VNM relation (see Eq. (10)). 


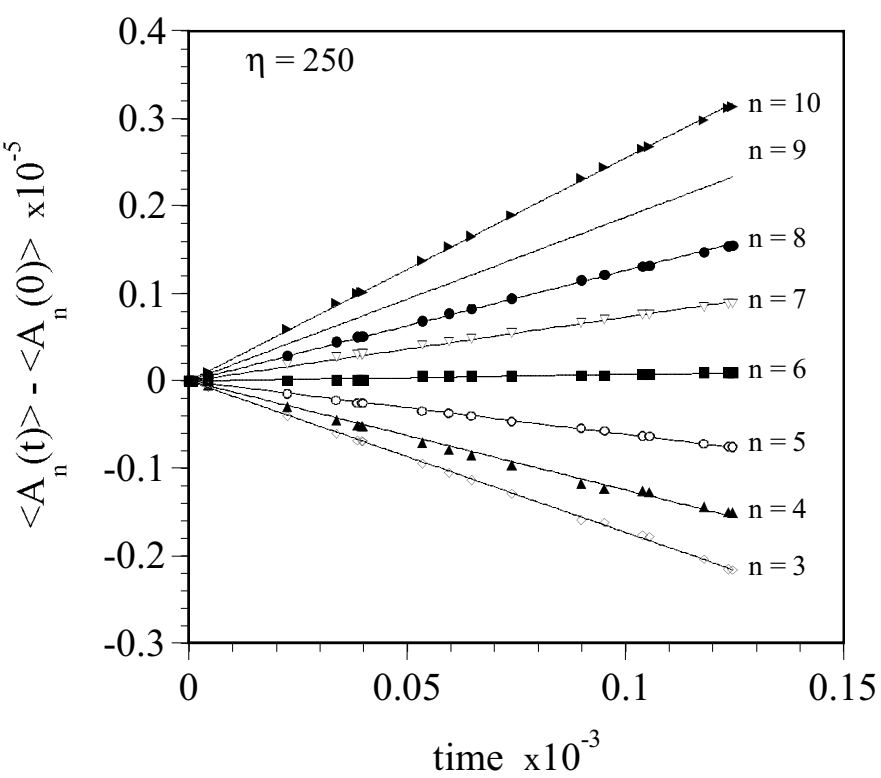

Fig. 8 Time evolution of the average grain area, $\left\langle A_{\mathrm{n}}(t)>-<A_{\mathrm{n}}(0)>\right.$, of $n$-sided grains for $\eta=250$. Only grains that do not change their number of sides during the entire time interval of the averaging are used when calculating $\left\langle A_{n}(t)>\right.$.

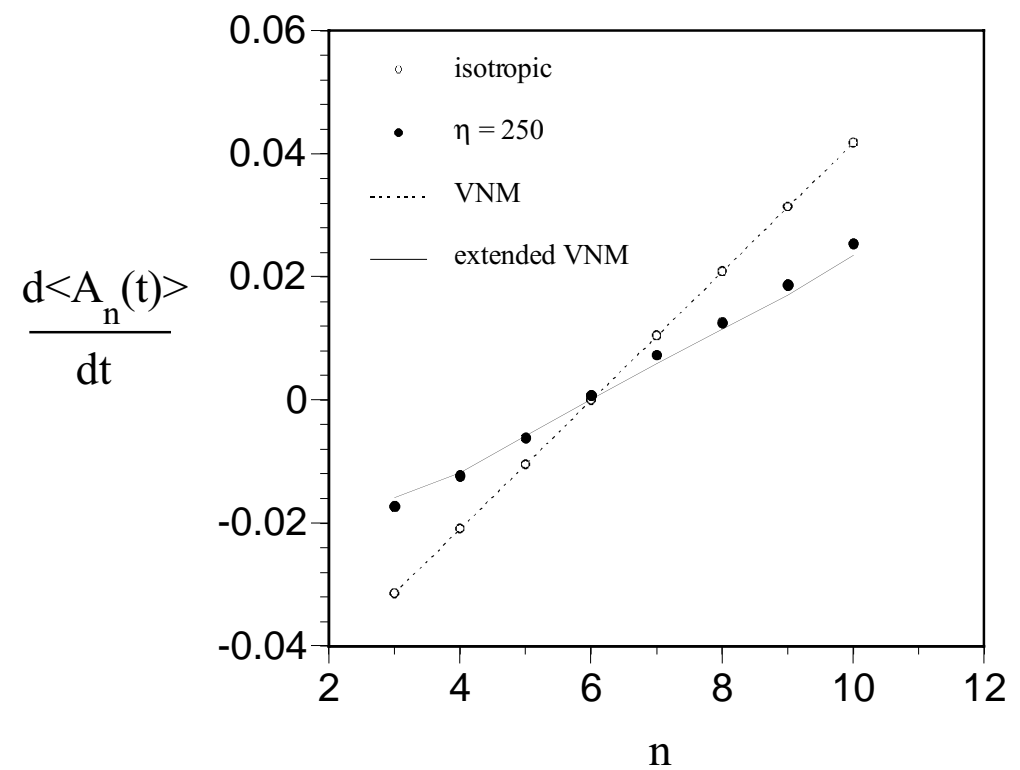

Fig. 9 Growth rates of the average areas of $n$-sided grains for the isotropic system and for the system with $\eta=250$. The simulation results are well represented respectively, by the von Neumann-Mullins relation (Eq. (10); dotted line) and by the extended von Neumann-Mullins relation (Eq. (11); solid line).

Figure 9 shows our simulation results for the rates of area change for grains belonging to various topological class $n$, for both the isotropic system and for a system with $\eta=250$. In addition, the rates predicted from Eq. (10) and from the extended averaged VNM relation, Eq. (11), are shown. As we saw previously [16], the VNM relation is followed almost perfectly for the isotropic case. Although the average rate of grain-area change shows some deviation from linearity and is not at all represented by the VNM relation, for $\eta=250$ the extended averaged VNM relation fits the simulation results well. This demonstrates that, although the degree of complexity increases in the simultaneous presence of anisotropic GB properties and grain 
rotation, the growth of the grains which are not involved in any grain coalescence events can still be rationalized in terms of groups of grains belonging to the same topological class.

\section{CONCLUSIONS}

By studying the grain growth in a $2 \mathrm{D}$ system in the presence of both GB migration and grain rotation, our mesoscopic simulations shows the presence of a physical length scale $R_{c}$. This enables the growth process to be characterized by two regimes. If the average grain size is smaller than $\mathrm{R}_{\mathrm{c}}$, grain growth is dominated by the grain-rotation-coalescence mechanism. By contrast, if the average grain size is greater than $R_{c}$, the growth is dominated by curvature-driven GB migration.

For the system evolving in the grain-rotation dominated regime we have found that the growth exponent, $n=2 /(p-1)$ is universal and depends only on one parameter, $p$, controlling size dependence of the grain rotational mobility. Although the degree of complexity of grain growth increases in the presence of both GB migration and grain rotation, not all the growth characteristics are changed. As our simulations show, the time evolution of the system is still governed by a power law. Moreover, our study also shows that an extended von NeumannMullins relation, based on averaged grain-boundary properties, can be further extended to include the effect of grain rotations.

As it was mentioned in in Sec. 4.1, the order-of-magnitude estimate based on the simulated GB mobility in a pure material yields a lower limit for $R_{c}$ of a few nanometers. because GB migration can be strongly impeded by impurities swept up and dragged along by a moving GB, experimentally determined GB mobilities can be orders of magnitude lower than the values extracted from simulations [1]. Moreover, a low mobility of the triple junctions would also significantly slow GB migration. As a consequence, according to Eq. (9), a lower value of GB mobility would significantly increases the physical length scale Rc, with the potential of bringing it into the micron grain-size range. In practice, the effect of grain rotations on grain growth may therefore not be restricted to nanocrystalline materials and may be important even for some coarse-grained materials.

\section{ACKNOWLEDGMENTS}

This work was supported by the U.S. Department of Energy, Office of Science, under Contract W-31-109-Eng-38.

\section{APPENDIX}

Growth law due to grain-rotation coalescence alone

Consider a two-dimensional system consisting of a large number, $\mathrm{N}$, of grains characterized by their orientation angles with respect to an arbitrary fixed direction. Each grain is free to rotate about an axis perpendicular to the plane in the clockwise or counterclockwise direction. The GB energy depends on the misorientation angle between any two neighboring grains and the change of the orientation of one grain due to rotation leads to a change in the misorientations of all the GBs surrounding a given grain. Consequently the energies of all the GBs surrounding the grain change such that overall the total energy of all the GBs delimiting the grain decreases. When two neighboring grains assume the same orientation, they coalesce forming a single larger grain. 
The total number of grains present in the system, $N$, decreases discontinuously every time a coalescence event takes place. By analogy with radioactive decay, we assume that grains can be characterized by some average "lifetime", $t_{L}$, equal to the average time it takes for a grain to undergo a coalescence event. Therefore we write

$$
\frac{1}{N} \frac{d N}{d t}=-\frac{1}{t_{L}}
$$

As two grains coalesce when they have acquired the same orientation, it is reasonable to assume that coalescence events take place with a frequency proportional to the average rate of grain rotation, $\langle\omega\rangle$. Therefore, $t_{\mathrm{L}}$ is proportional to $1 /<\omega>$.

Without loss of generality we assume that the $\mathrm{N}$ grains are located in a square box of size $\mathrm{L}_{\mathrm{b}}$ surrounded by periodic repliças in both directions. The average grain area, $A(t)$, is related to the total number of grains, $N(t)$, by $N(t) A(t)=L_{\mathrm{b}}$. By differentiating this relation, we find $N(t) \mathrm{d} A(t)+\mathrm{d} N(t) A(t)=0$. This is equivalent to $\mathrm{d} A(t) / A(t)=-$ $\mathrm{d} N(t) / N(t)$ which, by using Eq. (A.1), gives:

$$
\frac{1}{A} \frac{d A}{d t}=B_{1}^{-1}<\omega>
$$

where $\mathrm{B}_{1}$ is a constant. Thereby relating the rate of change of the average grain area, $\mathrm{d} A / d t$, to $\langle\omega\rangle$.

To integrate Eq. (A.2) we need to know the dependence of $\langle\omega\rangle$ on $A$. For an individual grain, $i, \omega_{\mathrm{i}}$ is given by Eq. (2) in which both the rotational mobility, $M$, and the cumulative torque, $\tau$, depend on the average grain size (see Eqs. (1) and (3)). For simplicity we assume that the average grain size $R$ is related to the average grain area $A$ by the relation $R=A^{1 / 2}$. We can define for each grain $i$ the aggregate GB energy gradient as $<\mathrm{d} \gamma / \mathrm{d} \theta>_{\mathrm{i}}=\left(1 / \mathrm{N}_{\mathrm{GB}}\right) \sum_{\mathrm{j}=1} \mathrm{~d} \gamma_{\mathrm{j}} / \mathrm{d} \theta_{\mathrm{j}}$, where $j$ runs over all the $\mathrm{N}_{\mathrm{GB}}$ GBs delimiting grain $i$. By averaging this over the whole system, we obtain

$$
\left\langle\frac{d \gamma}{d \theta}\right\rangle=\frac{1}{N} \sum_{i=1}^{N}\left\langle\frac{d \gamma}{d \theta}\right\rangle_{i}
$$

Here we assume, based on the simulations that during the growth $\langle\mathrm{d} \gamma / \mathrm{d} \theta>$ depends on the average grain area, $A$, as a power law, i.e., $<\mathrm{d} \gamma / \mathrm{d} \theta>\sim A^{\mathrm{q}}$. Using Eqs. (A.3) and (1), the average cumulative torque $\tau$ acting on a grain becomes

$$
\tau=B_{2} R\left\langle\frac{d \gamma}{d \theta}\right\rangle=B_{2} A^{1 / 2} A^{q}=B_{2} A^{(1+2 q) / 2}
$$

where $B_{2}$ is another constant. Inserting this together with Eq. (A.4) into Eq. (2) we see that the dependence of $\langle\omega>$ on $A$ is given by

$$
<\omega>=\frac{C}{A^{p / 2}} B_{2} A^{(1+2 q) / 2}=\frac{\text { const. }}{A^{(p-1-2 q) / 2}} .
$$

Inserting Eq. (A.5) into Eq. (A.2) and integrating for the time dependence of $A(t)$, we obtain:

$$
A(t) \sim t^{\frac{2}{p-1-2 q}}=t^{n^{\prime}}
$$

with growth exponent $n^{\prime}$ given by

$$
n^{\prime}=\frac{2}{p-1-2 q}
$$

Although n' depends on the two parameters $\mathrm{p}$ and $\mathrm{q}$, our simulations presented below show that $\mathrm{q}$ is very small. In fact, in the steady-state self-similar growth regime one can expect that $\langle\mathrm{d} \gamma / \mathrm{d} \theta>$ assumes a constant value, i.e., $\mathrm{q}=0$ (see Eq.(A.4)). This suggest that the growth exponent in the steady-state regime is given by 


$$
n=\frac{2}{p-1}
$$

As a limiting case, for $\mathrm{p}=1$ and assuming $\mathrm{q}=0$, Eqs. (A.2) and (A.5) predict an exponential increase in time for $\mathrm{A}(\mathrm{t})$.

\section{REFERENCES}

1. Humphreys, F.J., and Hatherly, M., Recrystallization and Related Annealing Phenomena, Pergamon, Elsevier Science Ltd., Oxford, 1995.

2. Burke, J. E. and Turnbull, D., Prog. Metal Phys., 1952, 3, 220.

3. Hillert, M., Acta metall., 1965, 13, 227.

4. Louat, N.P., Acta metall., 1974, 22, 721.

5. Mullins, W.W., J. Appl. Phys., 1986, 59, 1341.

6. Abbruzzese, G. and Lucke, K., Acta metall., 1986, 34, 905.

7. Grest, G.S., Srolovitz, D.J. and Anderson, P.M., Acta metall., 1985, 33, 509.

8. Rollett, A.D., Srolovitz, D.J., and Anderson, P.M., Acta metall., 1989, 37, 1227.

9. Frost, H.J., Thompson, C.V., Howe, C.L., and Whang, J., Scripta metall., 1988, $22,65$.

10. Maurice C. and Humphreys F. J., Grain Growth in Polycrystalline Materials, Proc. 3rd Int. Conf. 1998, eds. Weiland, H., Adams, B.L. and Rollett, A.D., TMS Publ., Warrendale PA, 1998, p. 81.

11. Kawasaki, K., Nagai, T. and Nakashima, K., Phil. Mag. B, 1989, B60, 399.

12. Weygand, D., Brechet, Y. and Lepinoux, J., Phil. Mag. B, 1998, B78, 329.

13. Frost, H.J., Thompson, C.V., Howe, C.L., and Whang, J., Scripta metall., 1988, $22,65$.

14. Cocks, A.C.F., and Gill, S.P.A., Acta mater., 1996, $44,4765$.

15. Gill, S.P.A., and Cocks, A.C.F., Acta mater., 1996, $44,4777$.

16. Moldovan, D., Wolf, D., Phillpot, S.R., and Haslam, A.J., Phil. Mag. A, 2002, 82, 1271.

17. Gottstein, G., and Shvindlerman, L.S., Scripta metall mater.,1992, 27, 1521.

18. von Neumann, J., in Metal Interfaces, ASM, Metals Park, Cleveland, Ohio, 1952, p. 108.

19. Mullins, W. W., J. Appl. Phys., 1956, 27, 900.

20. Herrmann, G., Gleiter, H. and Baro, G., Acta metall., 1976, 24, 353.

21. Lojkowski, W., Gleiter, H. and Maurer, R. , Acta metall., 1988, 36, 69.

22. Mykura, H., Acta metall ., 1979, 27, 243.

23. Ringer, S. P., Li, W. B. and Easterling, K. E., Acta metall. mater., 1992, 40, 275.

24. Harris, K.E., Singh, V.V. and King, A.H., Acta mater., 1998, 46, 2623.

25. Nichols, C.S., Mansuri, C.M., Townsend, S.J. and Smith, D.A., Acta metall. mater., 1993, 41, 1861.

26. Randle, V., Phil. Mag. A, 1993, 67, 1301.

27. Randle, V., Mat. Sci. \& Tech., 1991, 7, 985.

28. Yamasaki, T., Demizu Y. and Ogino, Y., Mater. Sci. Forum, 1996, 204-206, 461.

29. Haslam, A.J., Phillpot, S.R., Wolf, D., Moldovan, D. and Gleiter, H., Mat. Sci. and Eng. A, 2001, 318, 293.

30. Nichols, C.S., Cook, R.F., Clarke, D.R. and Smith, D.A., Acta metall. mater., 1991, 39, 1657.

31. Nichols, C.S., Cook, R.F., Clarke, D.R. and Smith, D.A., Acta metall. mater., 1991, 39, 1667.

32. Saetre, T.O., Ryum, N. and Evangelista, E., Metall. Trans. A, 1991, 22A, 2257.

33. Saetre, T.O. and Ryum, N., Metall. Trans. A, 1995, 26A, 1687.

34. Li, J.C.M., J. Appl. Phys., 1962, 33, 2958 .

35. Doherty, R.D. and Szpunar, J. A., Acta metall., 1984, 32, 1789.

36. Ryum, N., Acta Metall., 1969, 17, 831.

37. Shewmon, P.G., Chap. 5 in Recrystallization, Grain Growth and Textures, ASM, Metals Park, OH, 1966, p. 165.

38. Moldovan, D., Wolf, D. and Phillpot, S.R., Acta mater., 2001, 49, 3521

39. Raj, R. and Ashby, M.F., Metall. Trans., 1971, 2 , 1113.

40. Needleman, A. and Rice, J. R., Acta metall.,1980, 28, 1315.

41. Cleri, F., Physica A, 2000, $282,339$. 
42. Read, W. T., and Shockley, W., Phys. Rev., 1950, 78, 275.

43. Wolf, D., Scripta metall., 1989, 23, 1713.

44. Humphreys, F. J., Grain Growth in Polycrystalline Materials, Proc. 3rd Int. Conf. 1998, eds.

Weiland, H., Adams, B.L. and Rollett, A.D., TMS Publ., Warrendale PA, 1998, p. 13.

45. Herring, C., Chapter 8 in The Physics of Powder Metallurgy, W. E. Kingston, ed., McGraw-Hill, New York, 1951, p. 143.

46. Weaire, D. and Kermode, J.P., Phil. Mag., 1983, B48, 245; ibid 1984, B50, 379.

47. Mackenzie, J. K. and Thomson, M. J., Biometrika, 1957, 44, 205.

48. Samajdar, I. and Doherty, R. D., Scripta metall. et mater., 1994, 31, 527. 